\title{
Distribución de las Funciones Retóricas de las Citas en los Apartados del Trabajo Fin de Máster Escrito en Español y en Inglés
}

\author{
Distribution of the Rhetorical Functions of the Citations in the Sections \\ of the Master's Thesis Written in Spanish and in English
}

\begin{abstract}
This study correlates with a research study published in the journal Pragmalingüistica, in which the contrastive use of the rhetorical functions of citations in master's theses in Spanish and English was studied. The main objective pursued by this new research is to determine which rhetorical functions are used most frequently in the different parts of a master's thesis and what these can accomplish in each part of the text. Following Petrić (2007), a computerized textual analysis of the rhetorical function of citation was used to study this phenomenon in a corpus of sixteen (16) theses of which eight (8) were written by American and eight (8) by Spanish postgraduates in their native language. With regards to cultural conventions, the results showed that when compared to native Spanish writers, the Americans students use the highest number of citations and write a relatively longer Introduction and Conclusion parts. The sets of functions are consonant with the rhetorical purpose of each part, which thus shows a close link between the citation and the structural organization of the academic text, despite the frequent use of Attribution (AT) in most parts of the thesis.
\end{abstract}

\section{Keywords}

discourse analysis; academic writing; rhetorical functions of citation; Intercultural Rhetoric; rhetorical organization; master's thesis

\section{Introducción}

El acto de la citación es una de las características textuales más distintivas del lenguaje académico, ya que la referencia explícita a las fuentes establece una relación directa entre el escrito o la investigación novedosa del autor y el contexto de conocimiento en que se enmarca dentro de la disciplina. Sin embargo, la elaboración colaborativa del saber que se origina a partir del establecimiento de estas redes intertextuales está sujeta a distintos tipos de variación en función de las convenciones de la disciplina, el género o la lengua y cultura en las que esta se produce (p. ej. Hyland 1999, 2000; Thompson/Tribble 2001; Fløttum et al. 2006; Mur Dueñas 2009; Dontcheva-Navratilova 2016).

Por otra parte, la cita adoptará en el texto diversas funciones retóricas según sean los propósitos retóricos para los que se emplea en cada una de las partes del texto académico en las que se inserta. De este modo, el escritor utiliza este recurso -no siempre de modo consciente (Harwood 2009) - con el fin de expresar una serie de intenciones que se supeditan a la interacción dialógica que establece con el lector, a partir de la cual trata de guiarle en la lectura de su obra, al igual que ocurre también con otros recursos metadiscursivos interpersonales similares empleados con esta misma finalidad (Hyland 2005, 2017). En este sentido, numerosos trabajos se han hecho eco de la relevancia de este recurso en la organización retórica de los escritos académicos (Swales 1990, 2004; Hyland 1999, 2000, 2002; Thompson 2001; Thompson/Tribble 2001; Petrić 2007; Samraj 2008, 2013; Sánchez-Jiménez 2012; Venegas et al. 2013; Nguyen/Pramoolsook 2015; Dontcheva-

\footnotetext{
* David Sánchez-Jiménez

Department of Humanities

New York City College of Technology, CUNY

DSanchezJimenez@citytech.cuny.edu
} 
Navratilova 2016). En la presente investigación, nos centramos en el estudio de este fenómeno en el contexto concreto de la investigación de posgrado. Además de los trabajos en los que se describe de manera general el uso de las citas en distintos apartados de los escritos producidos por estudiantes de segundo y tercer ciclo universitario (Samraj 2008, 2013; Mur Dueñas 2009; SolerMonreal/Gil-Salom 2011,2014), existen otros en los cuales se han analizado de manera específica las funciones retóricas que desempeñan las citas en la organización de las distintas secciones de la memoria de máster ${ }^{1}$ (Petrić 2007; Sánchez-Jiménez 2012; Nguyen/Pramoolsook 2015) o de la tesis doctoral (Thompson 2001). Los resultados de estos estudios mostraron la variación hallada en las funciones retóricas de las citas utilizadas en diferentes secciones del trabajo académico de posgrado. Sin embargo, en ninguna investigación precedente de la que tengamos constancia se llevó a cabo un análisis contrastivo en distintas lenguas del comportamiento de este recurso pragmalingüístico en los diferentes apartados de los géneros universitarios mencionados, aunque sí se hayan realizado investigaciones similares sobre otros elementos retóricos del discurso (Leki 1991; Connor 1996, 2002, 2011; Grabe/Kaplan 1996).

Por este motivo, este artículo tiene como propósito principal investigar este nicho de conocimiento inexplorado con el fin de mejorar la práctica escrita de los estudiantes universitarios cuando redactan sus textos en inglés o español. En concreto, mediante este trabajo se pretende facilitar el conocimiento del uso de las funciones retóricas de las citas y su distribución en las distintas secciones de la memoria de máster al estudiante de posgrado que redacta este tipo de texto en inglés o en español tanto en L1 como, especialmente, en L2. Este estudio viene a complementar una investigación previa publicada en la revista Pragmalingüistica (Sánchez-Jiménez 2018a) en la cual se analizaba el comportamiento de las funciones retóricas de las citas en un estudio contrastivo intercultural realizado con un corpus compuesto por memorias de máster escritas en español y en inglés. Con ese mismo corpus, aunque ampliando el alcance del estudio, en esta nueva investigación exploramos cómo dicho recurso discursivo interactúa con la organización retórica de los diferentes apartados incluidos en este tipo de texto. Para ello, se analizaron las categorías de las funciones retóricas de las citas utilizadas en cada una de las secciones de los trabajos de fin de máster escritos por 8 estudiantes españoles y 8 de nacionalidad estadounidense en su lengua materna. El trabajo pretende conocer si existen diferencias en el uso de las citas causadas por el contraste de la lengua materna utilizada por los grupos y por la formación recibida en escritura académica en ambas culturas, así como saber si la distribución de las funciones retóricas de las citas y la intencionalidad del escritor en cada parte de la memoria de máster varían en la producción del hablante español que escribe en su lengua materna con respecto al estudiante estadounidense que lo hace en inglés. En relación con esta segunda variable, cabe destacar que los informantes del grupo español no han recibido formación específica en la elaboración de las memorias (al menos no tenemos constancia de que existieran cursos específicos de escritura académica en las universidades en las que fueron defendidas las memorias de máster analizadas en este estudio entre los años 2001-2010), más allá de su propio interés por estudiar el modelo de otros textos similares ya publicados, ponerse en contacto con autores expertos o solicitar ayuda de sus directores de máster. Por el contrario, la preparación académica de los estudiantes anglosajones en este aspecto de la escritura es una práctica habitual en las universidades estadounidenses desde finales del pasado siglo (Kinkead 2001).

\section{Marco Teórico}

\subsection{Los Estudios Contrastivos de los Géneros Discursivos Académicos: la Memoria de Máster}

Los estudios del análisis del discurso iniciados en los años 60 reparaban en diversos rasgos formales del discurso utilizados tanto en contextos orales como escritos. Según Flowerdew

1 Por cuestiones estilísticas relacionadas con la repetición y la redundancia, en el artículo se utilizan de modo indistinto e intercambiable -sin matiz diferenciador alguno- los términos memoria de máster y trabajo de fin de máster. 
(2002: 1), el impulso mayor recibido por estos estudios respondía a la necesidad de formación en las habilidades de comunicación prácticas que debían adquirir los hablantes no nativos en su aprendizaje del inglés. En el terreno de la escritura, dichos estudios trataban de identificar el comportamiento distintivo de los rasgos lingüísticos de los estudiantes que producían sus textos en L2 en relación con los modelos de la L1 de los que partían inicialmente, por lo que la metodología empleada urgía a establecer análisis contrastivos entre los hablantes extranjeros y los nativos. Estos procedimientos dieron como resultado la génesis de una nueva comunidad investigadora que adoptó esta metodología contrastiva con el fin de explorar no solo el comportamiento de los rasgos lingüísticos diferenciadores en distintas lenguas, sino también de los elementos discursivos y pragmáticos que conforman los géneros, esto es, la Retórica Contrastiva (RC), en nuestros días evolucionada a Retórica Intercultural (Connor 2011). La RC supuso el primer intento desde la Lingüística Aplicada por explicar cómo se producía la escritura en una lengua extranjera. Del mismo modo, se inició la exploración del discurso oral y escrito desde una perspectiva intercultural (que comenzaba a tener en cuenta las prácticas y las conductas comunicativas al establecer relaciones entre distintas culturas). Desde esta disciplina, la pedagogía se orientaba hacia el desarrollo de habilidades que hicieran más efectivas las relaciones cognitivas, sociales y pragmáticas que se establecen entre personas de distinto origen cultural, entendiendo que diferentes culturas tienen patrones distintos a la hora de producir y descodificar un texto para su adecuada interpretación. Según la teoría de la RC, las convenciones culturales se transfieren desde la L1 a la escritura en la L2, causando principalmente interferencias a nivel discursivo en la organización del texto, cuyas desviaciones eran a menudo penalizadas en la clase de lengua por los docentes. La RC permite examinar y entender las causas que se ocultan detrás de dichas desviaciones para ofrecer soluciones que faciliten la transmisión y comprensión del mensaje de forma más efectiva a hablantes de otras culturas (Sánchez-Jiménez 2015).

Los trabajos pioneros de Kaplan $(1966,1978)$ en esta ciencia encontraron pronto eco dentro del contexto académico en los Estados Unidos (Santiago 1970; Montaño-Harmón 1991; Lux/Grabe 1991; Reid 1992). Con posterioridad, las investigaciones que sucedieron a estos primeros estudios han venido centrándose en géneros específicos y se han especializado ya en los años 90 en el análisis de las diferencias retóricas existentes entre las lenguas en relación con la organización de los textos y en la interacción con la audiencia, superando así una primera etapa en la que se priorizaba el análisis de los rasgos formales (Flowerdew 2002: 2; Carbonell-Olivares et al. 2009: 152).

Con este cambio de rumbo, que en palabras de Flowerdew (2002: 2) llega a su madurez con las publicaciones de Swales (1990) y Bhatia (1993), se iniciaron también los trabajos de carácter empírico en el análisis de géneros profesionales y académicos, al mismo tiempo que se generalizaron las investigaciones que pretendían dar respuesta a las necesidades planteadas por el uso y la didáctica de las lenguas con fines específicos (Connor 1996, 2002; Grabe/Kaplan 1996). Dentro de los estudios de género aplicados a la enseñanza de las lenguas de especialidad, se puso el foco en la estructura organizativa de los textos y se trataron de establecer mediante su contraste los márgenes que diferenciaban cada género, disciplina y lengua en su organización retórica (p. ej. Swales 1990, 2004; Bhatia 1993; Dudley-Evans 2000; Hyland 2000; Thompson 2001, 2005; Bunton 2002; Connor 2002; Flowerdew 2002).

La gran mayoría de estos trabajos se realizaron en el ámbito experto de conocimiento en la universidad y en el intercambio académico profesional, por lo que los géneros más estudiados fueron, al principio, los correspondientes a estos entornos, especialmente el artículo de investigación. Con posterioridad, a principios de los años 90, surgió un interés creciente por explorar las producciones orales y escritas de los estudiantes no expertos de posgrado, centrado en un primer momento en el género de la tesis doctoral (p. ej. Dong 1996; Thompson 2001, 2005; Bunton 2002; Swales 2004; Carbonell-Olivares et al. 2009) y, en menor medida, en la memoria de máster (p. ej. Dudley-Evans 1986, 1994; Petrić 2007; Samraj 2008; Jalilifar 2012; SánchezJiménez 2013, 2018a). 
La memoria de máster, el género del que nos ocupamos en este artículo, cumple el propósito general de demostrar la suficiencia investigadora del estudiante de posgrado ante un Tribunal encargado de valorar su trabajo. Esta situación de asimetría en la que se posiciona el candidato va a condicionar de manera relevante los instrumentos retóricos que emplea para comunicarse con su audiencia, así como con el resto de los autores mencionados en el texto. No se debe perder de vista tal objetivo al considerar este género discursivo, observado siempre desde la óptica de un producto preparado para la lectura pública ante un grupo de expertos, quienes, desde su posición de poder evalúan si el aspirante se adapta a las normas sociales (no sólo de escritura, sino también del grado de conocimiento que atesora en la materia de estudio, el manejo de los términos específicos, la organización de los contenidos, etc.) convenidas por la comunidad científica. Este trabajo carece, por lo tanto, de las pretensiones trascendentes que puedan albergar los investigadores expertos (Dudley-Evans 1994; Samraj 2008), quienes exponen sus ideas en otros géneros especializados (artículo de investigación, manual didáctico, artículo de divulgación, etc.) y se diferencia también de estos en la posición de autoridad que como expertos adoptan en sus textos. Es desde este marco concreto que investigamos las funciones retóricas que cumplen las citas en los distintos apartados del trabajo académico de fin de máster escrito en inglés y en español, obviando la bibliografía que se ocupa de los estudios contrastivos centrados en este aspecto en otros géneros académicos y profesionales (p. ej. Fløttum et al. 2006; Mayor Serrano 2006; Mur Dueñas 2009; Mansourizadeh/ Ahmad 2011; Soler-Monreal/Gil-Salom 2011, 2014; Dontcheva-Navratilova 2016).

\subsection{Los Estudios Lingüísticos sobre la Citación en el Contexto Académico: las Funciones Retóricas de las Citas}

A pesar de que el análisis de las citas se originó primeramente en las disciplinas de las Ciencias de la Información y la Sociología de la Ciencia (para una revisión general, véase White 2004; Nicolaisen 2007; Sánchez-Jiménez 2011) con la finalidad de evaluar el impacto cuantitativo que este elemento desempeña en la interacción científica, a lo largo de las últimas décadas muchos lingüistas de la tradición anglosajona han puesto de relieve la importancia que atesoran las citas dentro de la escritura académica (p. ej. Swales 1986, 1990; Thompson/ Ye 1991; Hyland 1999, 2000, 2002; Thompson/Tribble 2001; Petrić 2007; Samraj 2008, 2013; Harwood 2009), así como también sucedió posteriormente en los estudios hispánicos (p. ej. Vázquez 2001; Beke 2008; Mur Dueñas 2009; Gallardo 2010; Soler-Monreal/Gil-Salom 2011, 2014; Sánchez-Jiménez 2013, 2018a; Venegas et al. 2013). En el caso de la Lingüística, el análisis de las citas se ha centrado en descubrir las relaciones y los vínculos que se establecen entre el citador y el texto citado. Estos estudios fueron impulsados desde el análisis textual primero (p. ej. Swales 1986, 1990; Hopkins/ Dudley-Evans 1988; Thompson /Ye 1991) y, con posterioridad, desde el análisis del discurso (p. ej. Hyland 1999; Thompson/Tribble 2001; Fløttum et al. 2006; Petrić 2007; Samraj 2008, 2013; Soler-Monreal/Gil-Salom 2011, 2014; Sánchez-Jiménez 2013, 2018a; Dontcheva-Navratilova 2016). En el primero de los casos, el análisis se centraba en los aspectos más formales del texto, fijándose en la construcción sintáctica de la cita en la oración o dentro del párrafo. Desde la segunda perspectiva, sin embargo, el énfasis se ponía en la relación que la cita mantiene con el resto de los elementos del discurso, en cómo este recurso contribuye a su organización, y en las funciones retóricas que las citas cumplen en el texto, cuyas imbricaciones contribuyen a aumentar la dialogicidad en el escrito.

Como consecuencia de estas investigaciones sobre la citación, se ha destacado en numerosos trabajos la relevancia retórica que este recurso adquiere en la organización textual de los escritos académicos (p. ej. Thompson 2001; Petrić 2007; Samraj 2008, 2013; Sánchez-Jiménez 2012; Dontcheva-Navratilova 2016; Nguyen/Pramoolsook 2015). De acuerdo con esta perspectiva, las distintas funciones empleadas en el texto supondrían un fiel diagnóstico de la estructura retórica del escrito en el que estas se insertan. Esto se debe a que en cada sección del texto el uso de las funciones de las citas ha de adecuarse a las intenciones retóricas propuestas en cada parte del trabajo por el investigador (Hyland 2000: 40; Thompson 2005: 321; Petrić 2007: 249; Sánchez-Jiménez 2012: 137). Por este motivo, se considera como uno de los elementos retóricos 
principales e identificativos de la escritura académica en su dimensión dialógica, la cual encuentra su especificidad propia dentro de cada disciplina y del estilo personal que cada autor adopta dentro de esta. De este modo, su significación sobrepasa su funcionalidad de referencia de las obras consultadas, pues los textos científicos se elaboran teniendo en cuenta la variabilidad de la escritura académica en las correspondientes comunidades discursivas, lo que establece la filiación de las citas con una determinada disciplina a la que las convenciones de su uso deben adecuarse (Hewings et al. 2010: 102).

Si Swales (1986) y Hopkins/Dudley-Evans (1988) presentaban los resultados de sus estudios sobre las citas desde planteamientos lingüísticos en los textos profesionales elaborados en los años 80 , en los 90 los investigadores comenzaron a interesarse por las características de las funciones de las citas en los textos producidos por escritores no expertos, tanto en L1 (p. ej. Thompson 2001; Thompson/Tribble 2001; Samraj 2008, 2013; Gallardo 2010; Venegas et al. 2013) como en L2 (p. ej. Dong 1996; Petrić 2007; Wette 2010; Sánchez-Jiménez 2013, 2018a; Soler-Monreal/ Gil-Salom 2011; Harwood/Petrić 2012; Jalilifar 2012; Rabab'ah/Ahmed Al-Marshadi 2013). En el primer caso, las investigaciones ahondaban en las diferencias interdisciplinarias de las convenciones de las comunidades discursivas en la citación (Hyland 1999, 2000; Samraj 2008), las que suceden entre los géneros (Mayor Serrano 2004, 2006; Beke 2008) y las divergencias lingüísticas y culturales producidas en el uso de las citas en textos de distintas nacionalidades (Fløttum et al. 2006; Mur Dueñas 2009). Por otro lado, en la investigación realizada sobre el uso de las citas con alumnos universitarios, los estudios se han centrado en identificar las dificultades que los investigadores noveles encuentran en la realización de las citas, así como en probar la necesidad de la instrucción de este recurso lingüístico, las posibles mejoras que reporta su didáctica explícita y el modo más factible de llevarla a cabo (p. ej. Dong 1996; Petrić 2007; Wette 2010; Sánchez-Jiménez 2018b). Otros investigadores (Hyland 2002; Mansourizadeh/Ahmad 2011; Harwood/Petrić 2012; Jalilifar 2012; Rabab'ah/Ahmed Al-Marshadi 2013; Samraj 2013; Sánchez-Jiménez 2018c) han tratado de profundizar en el distinto uso que los escritores expertos y los inexpertos hacen de las citas mediante una comparativa de sus producciones.

Dentro de este marco, en un intento por contextualizar nuestro estudio, nos interesamos por las investigaciones que se han llevado a cabo contrastando diferentes lenguas y culturas, tanto las que existen en relación con el análisis de artículos de investigación escritos por expertos (Fløttum et al. 2006; Mayor Serrano 2006; Mur Dueñas 2009; Dontcheva-Navratilova 2016) como las que se refieren a los textos universitarios (Soler-Monreal/Gil-Salom 2011; Jalilifar 2012; Rabab'ah/ Ahmed Al-Marshadi 2013; Sánchez-Jiménez 2013, 2018a). Del mismo modo, se tuvieron en cuenta los abundantes trabajos realizados sobre las citas en el género de la memoria de máster desde las diferentes perspectivas -sintáctica, textual y retórica-discursiva- desde las que se ha abordado su estudio (p. ej. Petrić 2007; Samraj 2008, 2013; Jalilifar 2012; Rabab'ah/Ahmed AlMarshadi 2013; Sánchez-Jiménez 2013, 2018a; Venegas et al. 2013; Nguyen/Pramoolsook 2015).

\section{Metodología}

\subsection{Procedimiento}

Como ya se refirió en el artículo publicado en la revista Pragmalingüística (Sánchez-Jiménez 2018a), con el que la presente investigación comparte el corpus analizado, el estudio se compone de 16 memorias de máster escritas en español (e) y en inglés (i) en la disciplina de la Lingüística Aplicada. Se establecieron dos subcorpus divididos según la lengua, 8 textos producidos en español por hablantes nativos españoles (grupo E) y 8 en inglés compuestos por estudiantes estadounidenses (grupo A). Así, el informante 1 del grupo español, por ejemplo, fue codificado en el texto con la fórmula Ee1, que hace referencia al grupo de pertenencia (E), el idioma de producción (e) y el número que ocupa en el grupo (1). Un segundo tipo de codificación fue utilizado también con la finalidad de identificar los distintos apartados de la memoria de máster que se mencionan de manera frecuente en el cuerpo, las tablas y las figuras referidas a lo largo 
del texto: Introducción (INTR), Revisión de la literatura (MT), Metodología (MET), Resultados (RES), Conclusiones (CON) y Propuesta didáctica (PD). Sin embargo, antes de proceder a la descripción del análisis del corpus, es conveniente realizar algunas puntualizaciones sobre esta clasificación. En este sentido, solo aquellos apartados que han sido marcados con una signatura (INTR, MT, MET, RES, CON y PD) aparecen representados en las memorias analizadas, pues los escritores han prescindido en la mayoría de los casos, por ejemplo, del apartado de la Discusión, que en ocasiones aparece de forma explícita, aunque fusionado con el de las Conclusiones. Por lo tanto, hemos optado por homogeneizar los datos compilados en nuestro estudio seleccionando la etiqueta de la opción predominante, la de Conclusiones. Algo similar ocurre con las secciones de Metodología y Resultados, que en ocasiones aparecen unidas bajo el epígrafe Parte práctica o Análisis. En ambos casos se ha denominado este apartado como Metodología en el estudio, por ser el epígrafe que más se repite en las memorias analizadas. De tal modo, en estos ejemplos que se comentan ocurre que dos secciones que normalmente se presentan de forma independiente en los esquemas arquetípicos de la estructura de la investigación se combinan en una sola.

Con el fin de mantener la homogeneidad de los escritos -y para que estos resulten comparablesse controlaron algunas variables que permanecen constantes en todos ellos, como en el caso de la fecha de realización de los trabajos (escritos entre los años 2001 y 2010), la disciplina, el tema seleccionado, el sexo del autor, el número de palabras registrado por memoria y si fueron desarrollados y defendidos en universidades de España o Estados Unidos. El periodo temporal que abarca la defensa de los textos analizados en las universidades españolas es un factor relevante que condiciona la hipótesis de partida de esta investigación. A diferencia de lo que sucede en el momento presente - tras la revolución que supuso el plan Bolonia de 2007 en los programas de educación superior en España-, en el momento en el que fueron escritos estos textos, no existía una conciencia generalizada de la importancia de implementar una didáctica sobre las cuestiones relacionadas con la escritura académica en la universidad española y, más concretamente, en el trabajo de posgrado. Mientras que en países como los Estados Unidos estas prácticas se vienen realizando de modo estandarizado desde más de medio siglo (Kinkead 2001), en España su lenta incorporación curricular en el ámbito universitario ha sido más reciente.

En el análisis de los datos, se aplicó el método del análisis textual computarizado de las funciones retóricas de las citas en su contexto lingüístico (para una descripción pormenorizada de este proceso, véase Sánchez-Jiménez 2018a). Para ello, se tuvieron en cuenta tanto las etiquetas descriptivas de la funcionalidad de la cita (see, véase, vid., ej., etc.), como el significado de los verbos introductores de las citas y las palabras circundantes a estos. Los verbos introductores de las citas (decir, referir, postular, opinar, etc.) denotan la intencionalidad del autor al referir las fuentes en el texto, pues su selección supone una decisión retórica de carácter ilocutivo que conlleva claras consecuencias pragmáticas en la relación que se establece con el lector de la memoria de máster, factor este que viene a condicionar la manera en que se interpreta el escrito (Hyland 2000; Thompson, 2001; Sánchez-Jiménez 2018c).

Tras la clasificación de las citas, se procedió a su etiquetado y lematización en el texto mediante el programa Antconc 3.2.4w, con el fin de establecer la búsqueda y el cálculo de concurrencias. Con la ayuda de esta herramienta se realizó un cómputo de las citas más exacto y se revisitaron las concordancias en las que se encontraban las referencias analizadas. Finalmente, se calcularon las figuras agrupadas de los datos obtenidos multiplicando el número de las citas por $1.000 \mathrm{y}$ dividiendo el resultado por el total de palabras que contiene cada memoria de máster. Este es un procedimiento habitual en los estudios sobre las funciones de las citas, ya que permite comparar los resultados de los diferentes textos objeto de análisis.

\subsection{Tipología de las Funciones Retóricas de las Citas}

En este artículo, se ofrece una clasificación de las funciones retóricas de las citas de un modo simplificado y esquemático, en el que se reproducen de manera sintética las categorías que ya han sido explicadas de forma más detallada en otros trabajos precedentes, a los que se remite al 
lector que desee ampliar esta información (Sánchez-Jiménez 2011, 2012, 2013, 2018a). En ella, se distinguen 9 tipos diferentes de funciones retóricas de las citas:

\begin{tabular}{|c|c|c|}
\hline Tipo de Cita & Definición & Ejemplo \\
\hline $\begin{array}{l}\text { Atribución } \\
\quad \text { (AT) }\end{array}$ & $\begin{array}{l}\text { Tiene valor informativo y declara la } \\
\text { opinión, idea o proposición del autor } \\
\text { referido. }\end{array}$ & $\begin{array}{l}\text { It is important to acknowledge that "alignment is pro- } \\
\text { foundly multidimensional" (Atkinson et al., 2007, p. } \\
\text { 184), implying that there is no one correct way to ap- } \\
\text { proach the learning experience. (Ail-INTR). }\end{array}$ \\
\hline $\begin{array}{l}\text { Ejemplificación } \\
\text { (EJ) }\end{array}$ & $\begin{array}{l}\text { El autor ilustra una afirmación aludi- } \\
\text { endo a la fuente. }\end{array}$ & $\begin{array}{l}\text { Adjuntamos un ejemplo de visualización para relajar a } \\
\text { nuestros alumnos antes de comenzar la clase (Arnold, } \\
\text { 1999:283): (Ee1-MT). }\end{array}$ \\
\hline $\begin{array}{l}\text { Referencia } \\
\text { (REF) }\end{array}$ & $\begin{array}{l}\text { Se redirige al lector al trabajo men- } \\
\text { cionado para ampliar la información. }\end{array}$ & $\begin{array}{l}\text { A research goal of other existing studies has been to com- } \\
\text { pare native and non-native speakers of American English } \\
\text { on their attitude toward American English slang (see } \\
\text { Charkova, } 2007 \text { for a review) (Ai2-MT). }\end{array}$ \\
\hline $\begin{array}{l}\text { Declaración de } \\
\text { uso (DE) }\end{array}$ & $\begin{array}{l}\text { Declara que el trabajo citado o una de } \\
\text { sus partes se ha utilizado en el texto } \\
\text { presente. }\end{array}$ & $\begin{array}{l}\text { La clasificación según la forma del input responde a la } \\
\text { tipología propuesta por Dominique Macaire y Wolfram } \\
\text { Hosch, recogida por Ch. Cuadrado, Y. Díaz y M. Martín } \\
\text { (1999) (Ee4-MET). }\end{array}$ \\
\hline $\begin{array}{l}\text { Aplicación } \\
\text { (AP) }\end{array}$ & $\begin{array}{l}\text { Establece conexiones entre el citador } \\
\text { y el trabajo citado sobre algún con- } \\
\text { cepto, argumento, terminología o pro- } \\
\text { cedimiento usado en el trabajo citado. }\end{array}$ & $\begin{array}{l}\text { The foundation for my thesis is based on Vygotsky's } \\
\text { (1978) theoretical concept of the zone of proximal de- } \\
\text { velopment (ZPD), which will be explained in further de- } \\
\text { tail in the literature review (Ail-INTR). }\end{array}$ \\
\hline $\begin{array}{l}\text { Evaluación } \\
\quad(E V)\end{array}$ & $\begin{array}{l}\text { Se realizan valoraciones sobre un tra- } \\
\text { bajo precedente. }\end{array}$ & $\begin{array}{l}\text { Junto a las innegables virtudes que atesora, esta investi- } \\
\text { gación [S. Fernández] oculta en mi opinión algunos de- } \\
\text { fectos de naturaleza metodológica que merecen atención } \\
\text { (Ee6-MT). }\end{array}$ \\
\hline $\begin{array}{l}\text { Enlaces entre } \\
\text { las fuentes (EN) }\end{array}$ & $\begin{array}{l}\text { Señala enlaces, comparaciones o con- } \\
\text { trastes entre las fuentes usadas. }\end{array}$ & $\begin{array}{l}\text { While Krashen (1985) thought comprehensible input was } \\
\text { the major condition necessitating SLA, Swain (1995) hy- } \\
\text { pothesized that in the production of language a learner } \\
\text { must consider Syntax (Ai4-MT). }\end{array}$ \\
\hline $\begin{array}{l}\text { Comparación } \\
\text { de los resulta- } \\
\text { dos }(\mathrm{CO})\end{array}$ & $\begin{array}{l}\text { Indica similitudes o diferencias con } \\
\text { los trabajos del autor citado en el } \\
\text { apartado en el que se comparan los } \\
\text { resultados de la investigación. }\end{array}$ & $\begin{array}{l}\text { Confirmamos con estas cifras la observación de Ortega } \\
\text { Masagué (2005: } 6 \text { \& 9) citada en la revisión bibliográfica } \\
\text { (Ee3-RES). }\end{array}$ \\
\hline $\begin{array}{l}\text { Competencia } \\
\text { (COMP) }\end{array}$ & $\begin{array}{l}\text { Concentra una lista de citas de varios } \\
\text { autores en torno a una afirmación. }\end{array}$ & $\begin{array}{l}\text { Reading supplies the learners an enormous amount of in- } \\
\text { formation that broaden their perspective and eventually } \\
\text { improve their language performance particularly in pro- } \\
\text { ducing written discourses (Rivers, 1978; Reinking, Hart, } \\
\text { and von der Ostern, 1988; Harris, 1993) (Ai4-CON). }\end{array}$ \\
\hline
\end{tabular}

Tabla 1: Tipología de las funciones retóricas de las citas.

La tipología de las funciones retóricas de las citas de la que se parte en el presente estudio es una adaptación de la taxonomía establecida por Petrić en su investigación de 2007, realizada con estudiantes centroeuropeos en la disciplina de los Estudios de Género. Esta es una de las más de 40 calificaciones disponibles que se han desarrollado dentro de esta tradición, las cuales fueron creadas en función de las perspectivas que a cada autor más le interesaba de acuerdo con las convenciones vigentes en su disciplina y la metodología específica empleada en su estudio. La que ofrece Petrić (2007), con sus eventuales modificaciones (explicadas en Sánchez-Jiménez 2011, 2013, 2018a), se adecúa perfectamente al diseño de la presente investigación.

\section{Resultados y Discusión}

\subsection{Resultados Globales}


En las 16 memorias de máster analizadas, se registraron 2.653 citas, 1.042 de ellas emitidas por el grupo español y 1.611 por el estadounidense. Los resultados globales ${ }^{2}$ de la investigación expuestos en la Tabla 2 muestran una primera diferencia relevante basada en el número de las citas resultante en cada grupo. El empleo más elevado de este recurso por parte de los estudiantes estadounidenses adquiere mayor significación cuando se observa que la longitud de sus textos en número de palabras es menor. Esto supone que un estudiante de nacionalidad española utiliza una cita por cada 208,05 palabras, mientras que la frecuencia de aparición en las memorias producidas por estudiantes estadounidenses es significativamente mayor, una por cada 116,45 palabras.

\begin{tabular}{|lc|c|c|c|c|}
\cline { 3 - 5 } \multicolumn{1}{c|}{} & Número de Citas & $\begin{array}{c}\text { Número de } \\
\text { Palabras por } \\
\text { Memoria }\end{array}$ & $\begin{array}{c}\text { Cita por Número de } \\
\text { Palabras }\end{array}$ & $\begin{array}{c}\text { Citas por Cada } \\
\text { 1.000 Palabras }\end{array}$ \\
\hline Grupo Ee & 1.042 & 216.797 & 208,05 & 4,80 \\
Grupo Ai & 1.611 & 187.672 & 116,49 & 8,58 \\
& Total & 2.653 & 404.469 & 152,45 & 6,55 \\
\hline
\end{tabular}

Tabla 2: Datos globales sobre las memorias de máster escritas en español y en inglés.

La cuarta columna de la Tabla 2 refleja la medida de densidad del número de citas producidas por cada 1.000 palabras, la cual permite comparar los resultados obtenidos entre los grupos y también con otros estudios realizados sobre las funciones de las citas (que también utilizan esta medida de forma estandarizada). De acuerdo con los resultados obtenidos en nuestra investigación, se aprecia que el grupo norteamericano casi dobla al español en la producción de citas. Este dato coincide con los estudios previos que se han llevado a cabo en este ámbito entre ambas lenguas (p. ej. Mayor Serrano 2006; Mur Dueñas 2009; Soler-Monreal/Gil-Salom 2014).

\subsection{Resultados Globales sobre la Frecuencia de las Citas por Sección}

Como paso previo al análisis del número de citas que producían los escritores en cada apartado del trabajo de fin de máster y de las funciones que estas cumplen en el texto, conviene informar al lector sobre las peculiaridades que presentan las secciones de los escritos del corpus compilado para esta investigación. Tales cuestiones tienen repercusión en la variación de los resultados cuantitativos obtenidos en el estudio, ya que no todos los informantes incluyen el mismo número de secciones en sus textos ni los elaboran de la misma manera. De este modo, mientras que la superestructura de las memorias de máster resulta más homogénea en el grupo Ai, la diversidad es la característica definidora de la superestructura en Ee. El factor principal que justifica los diferentes resultados obtenidos en este aspecto discursivo del estudio puede relacionarse con la formación recibida por los escritores norteamericanos en cursos específicos de escritura académica, en los que aprendieron temas relacionados con la estructuración y la organización retórica de este y otros tipos de texto. Además, los estudiantes universitarios en este país cuentan en sus universidades con Centros de Escritura donde reciben formación adicional sobre esta materia, los cuales brindan al alumno la posibilidad de interactuar con tutores especializados para resolver las dudas que puedan surgir durante el proceso de elaboración de los distintos géneros discursivos estudiados en este nivel académico. ${ }^{3}$

2 Con el fin de evitar la constante interrupción de la lectura durante la exposición de los resultados, a partir de la Tabla 2 solo figurarán en el cuerpo del trabajo los datos numéricos más relevantes necesarios para comprender el estudio y glosar los gráficos que ilustran de manera representativa los resultados. Las tablas correspondientes a estos elementos pueden consultarse en Anexo I y Anexo II.

3 Prueba de la existencia de esta pedagogía es la tercera edición del manual de escritura académica superventas publicado por primera vez por Swales y Freak en 1994. El libro contiene explicaciones y estrategias dirigidas al lector para asistirle en la redacción de sus trabajos universitarios, mejorar la escritura académica y facilitar la didáctica a hablantes nativos y extranjeros que pretenden adaptarse a las convenciones textuales del sistema educativo superior de los Estados Unidos. 
Por el contrario, la irregularidad en la estructuración de la memoria de máster es evidente en los textos escritos en español, como se refleja en la Figura 1, posiblemente debido a la falta de instrucción en escritura académica de la que adolecían los estudiantes universitarios españoles en el periodo estudiado (2001-2010). En este esquema, se observa cómo los escritores españoles prescinden de secciones esperables en un trabajo de investigación de posgrado, como los apartados de Metodología, Resultados o Conclusiones (En parte, esto se debe a la combinación de dos de estos apartados en uno solo, como se explicó en detalle más arriba, § 3.1.).

\begin{tabular}{|c|c|c|c|c|c|c|c|c|c|c|c|c|c|}
\hline & INTR & MT & MET & RES & PD & CON & & INTR & MT & MET & RES & PD & CON \\
\hline Eel & & & & & & & $A i 1$ & & & & & & \\
\hline Ee2 & & & & & & & $A i 2$ & & & & & & \\
\hline Ee3 & & & & & & & $A i 3$ & & & & & & \\
\hline Ee4 & & & & & & & Ai4 & & & & & & \\
\hline Ee5 & & & & & & & $A i 5$ & & & & & & \\
\hline Ee6 & & & & & & & Ai6 & & & & & & \\
\hline $\mathrm{Ee} 7$ & & & & & & & $A i 7$ & & & & & & \\
\hline Ee8 & & & & & & & Ai8 & & & & & & \\
\hline
\end{tabular}

Figura 1: Esquema de las secciones en las memorias de máster analizadas.

En la Figura 2, se aprecia gráficamente el porcentaje de las citas producidas por sección en el total de las memorias analizadas. La sección en la que se realiza un mayor número de citas por cada 1.000 palabras es MT, con un 49\%, lo que prácticamente representa la mitad sobre el total de las referencias utilizadas en el corpus. También es numerosa la cantidad de citas contenidas en el apartado de INTR, con un $25 \%$. Combinadas, ambas secciones juntas concentran casi las tres cuartas partes del total de las citas empleadas en el corpus (74\%). Esto se debe, sin duda, a la relevancia que poseen ambos apartados en la contextualización del estudio, así como en la definición de los conceptos teóricos que se analizan en el trabajo fin de máster dentro de la disciplina de la Lingüística Aplicada. El resto de las secciones representa una densidad relativa en el número de las citas (MET y RES, ambas con un 8\% y CON, que suma el 9\%). Una mención aparte merece el apartado PD por la anomalía que supone su uso en uno de los subcorpus analizados. Tal desviación se debe a la omisión de este apartado de forma generalizada en los trabajos escritos por estudiantes estadounidenses. Parece ser habitual que, en la disciplina de la Lingüística Aplicada en la universidad española, estos textos incluyan una aplicación didáctica relacionada con los resultados obtenidos en la investigación teórica o empírica realizada en el estudio. Este género parece estar más orientado allí a la explotación didáctica de los resultados obtenidos en la investigación, mientras que en el contexto estadounidense cuenta con esquemas más fijos, más estandarizados y que obvian este cometido pedagógico para centrarse en los resultados obtenidos en el estudio. Sobre esto, Swales (2004: 99-100) comenta que la memoria de máster escrita por estudiantes estadounidenses en lengua inglesa parte del objetivo de solucionar problemas concretos identificados en una actividad o campo del saber, lo que la diferencia de otros géneros como, por ejemplo, la tesis doctoral. Por lo tanto, parece que el motivo principal que anima a los escritores (Ee3, Ee6, Ee7, Ee8, Ai5) a incluir una propuesta didáctica en su memoria de máster se explica por el propósito con el que se concibe este género en su tradición cultural. 


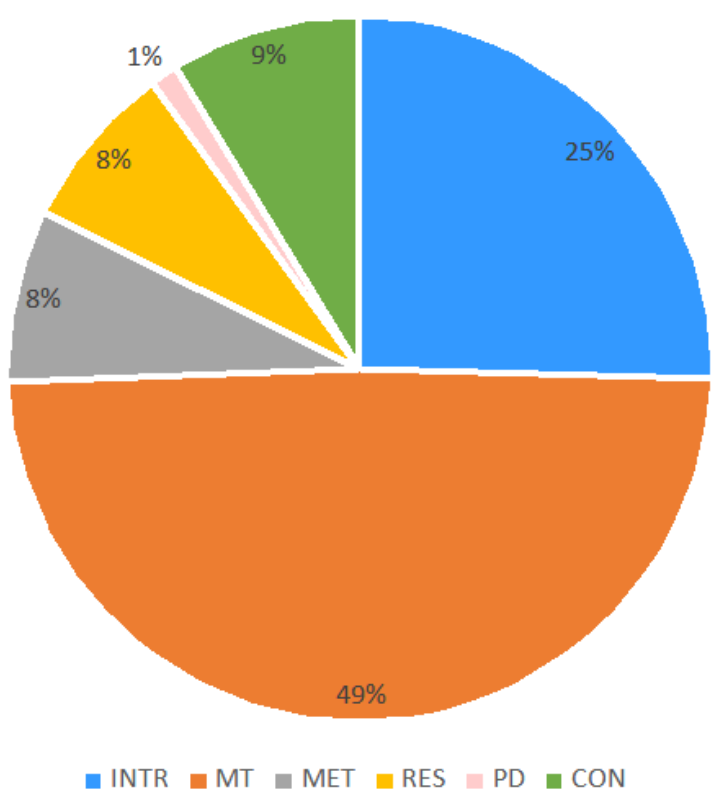

Figura 2: Porcentaje de la densidad de citas por sección.

En cuanto al cómputo de citas en este apartado, conviene señalar que las actividades $-\mathrm{y}$ las correspondientes explicaciones pedagógicas- que suelen formar parte de la sección no incluyen normalmente un elevado número de citas ( $1 \%$ sobre el total), aunque, en ocasiones, el número de palabras resulte tanto o más extenso que la propia investigación, lo que, a su vez, justifica la baja densidad de las citas registradas en PD.

\subsection{Resultados del Contraste entre los Grupos sobre la Frecuencia de las Citas por Sección}

En cuanto a los resultados comparativos de las citas emitidas por los grupos en cada sección, estas se presentan ordenadas por la densidad de las cifras agrupadas en la Figura 3 (expuestas en los Anexos I y II). Estos datos revelan que la sección que contiene una mayor cantidad de citas por número de palabras en ambos grupos, con amplia distancia del resto, es MT, seguida de INTR, tal y como sucedía también en el análisis de los resultados globales. Sin embargo, si se comparan entre sí las cifras por sección de manera pormenorizada, en este caso se aprecia que existe una diferencia significativa en la densidad de las citas producidas por cada grupo. Así, en MT, el subcorpus Ai $(18,48)$ dobla las cifras obtenidas en Ee $(9,15)$, mientras que en INTR estas se triplican (Ai: 11,17; Ee: 3,19). Esta constante se mantiene también en el resto de las secciones, aunque las diferencias no son tan elevadas como en estos apartados, excepto en el 
caso de CON, en el que las cifras vuelven a triplicarse (Ai: 3,85; Ee: 1,08). De esta divergencia se deduce que las memorias escritas en español cuentan con peculiaridades discursivas propias que las diferencian de las redactadas en inglés, pues estos escritores apenas aportan referencias a las fuentes consultadas en los apartados mencionados.

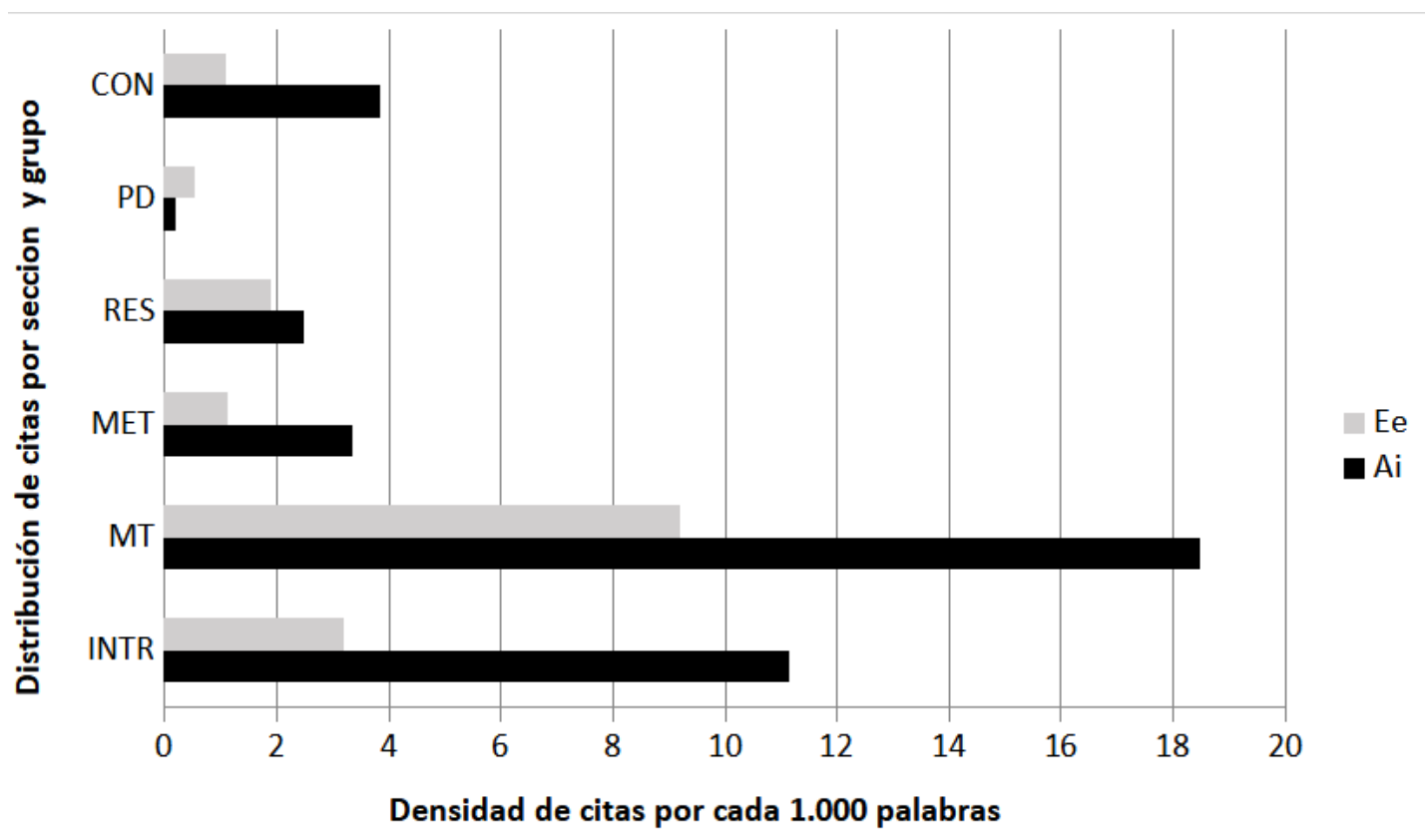

Figura 3: Densidad de las citas por sección en las memorias escritas en español e inglés.

En RES y MET, varían las cifras entre Ai y Ee, pero esto puede deberse a la fluctuación causada por la combinación de los apartados RES + Discusión y CON + Discusión, lo cual, en cierta medida, invalida la comparación de estas secciones en el corpus estudiado (§ 3.1.). Algo parecido sucede en el apartado PD con la cifra aislada de 0,20 emitida por Ai5 en el grupo norteamericano, ya que esta desviación de los datos causada por la producción de un único informante desvirtúa los resultados totales del grupo e invalida su contraste. No obstante, como se comentó en el subapartado anterior (§ 4.2.), esta es la única sección en la que Ee computa una cifra más elevada de citas que Ai, debido a la propia idiosincrasia de las memorias de máster defendidas en este contexto cultural.

Por lo tanto, a la luz de los datos analizados, comprobamos que las diferencias más relevantes entre ambos grupos ocurren en las secciones INTR y CON, en las que los estudiantes españoles apenas hacen referencia a las fuentes consultadas en estas secciones, en claro contraste con el comportamiento del grupo Ai. De hecho, la mitad de los informantes de Ee (Ee2, Ee3, Ee4, Ee6) no cita a ningún autor en la sección INTR, mientras otros dos de los informantes (Ee7, Ee8) producen una escasa cantidad de citas en este apartado. Esta distinción revela un uso diferenciado del propósito retórico y de la organización de esta sección por parte de ambos grupos. Por un lado, los informantes de Ee utilizan este espacio para presentar el tema de la investigación, su 
relevancia, su justificación y, en ocasiones, la estructura de la obra. Sin embargo, obvian la definición de conceptos fundamentales utilizados en el estudio o el comentario de investigaciones previas realizadas en esta área de conocimiento, componentes retóricos estos de los que se ocupan con mayor detenimiento en la sección MT (Sánchez-Jiménez 2012). Por su parte, los estudiantes norteamericanos tienden a ofrecer en este apartado una visión de conjunto sobre la investigación realizada con el fin de contextualizar el tema tratado. En ocasiones, se definen los conceptos y los objetivos del estudio y también se comentan algunas de las obras principales consultadas para llevar a cabo la investigación, además de describir la estructura del trabajo.

Los datos a los que aludimos en INTR coinciden con los obtenidos por Soler-Monreal/Gil-Salom (2011) en un estudio similar realizado con respecto a las citas contenidas en 20 tesis doctorales escritas en inglés y en español. Las autoras encontraron que la mayor presencia y visibilidad que se le otorgaba a la propia investigación dentro de la comunidad científica que elaboraba sus textos en inglés se correspondía con el énfasis que ponen los autores anglosajones en delimitar el territorio, promocionar la originalidad del tema elegido en su investigación y posicionar la validez de su estudio con respecto a sus iguales en la disciplina. La importancia otorgada a promocionar el escrito propio persigue, por lo tanto, el objetivo de distinguir su obra del resto de los autores que han propuesto investigaciones similares dentro de su comunidad discursiva. Así lo describían también Soler-Monreal et al. (2011: 8) en otro trabajo con estudiantes de doctorado en el que comparaban el uso y la frecuencia de la citación en relación con la estructura retórica de los escritos de posgrado elaborados en inglés y en español en la misma disciplina.

Algo similar a lo apuntado en estos estudios en relación con la sección INTR sucede en el apartado CON en nuestra investigación, en la que cuatro informantes españoles no producen ninguna cita (Ee2, Ee3, Ee4, Ee6). Nuevamente, el propósito de CON en los textos de posgrado redactados en inglés parece entenderse de manera diferente en la cultura española. De modo parecido a lo comentado sobre la sección INTR, Fløttum et al. (2006), Mur Dueñas (2009) y SolerMonreal/Gil-Salom (2011) coinciden en identificar una actitud promocional generalizada en el apartado CON en los trabajos académicos escritos en lengua inglesa como consecuencia del alto grado de competitividad existente en el círculo científico anglosajón y a la internacionalización de las publicaciones compuestas en este idioma. Por ello, en estos estudios, los autores destacan los resultados obtenidos en su investigación con respecto a los de los otros trabajos similares citados en ese mismo contexto temático.

\subsection{Resultados del Contraste entre los Grupos sobre la Frecuencia de las Funciones Retóricas de las Citas por Sección}

En este apartado se expone la información relativa a la frecuencia con la que cada grupo utiliza las funciones retóricas de las citas en cada una de las secciones de las memorias de máster analizadas. Las Figuras 4 y 5 exponen los datos recogidos en el análisis de las memorias escritas en lengua española por el grupo Ee y en inglés por estudiantes de Ai respectivamente.

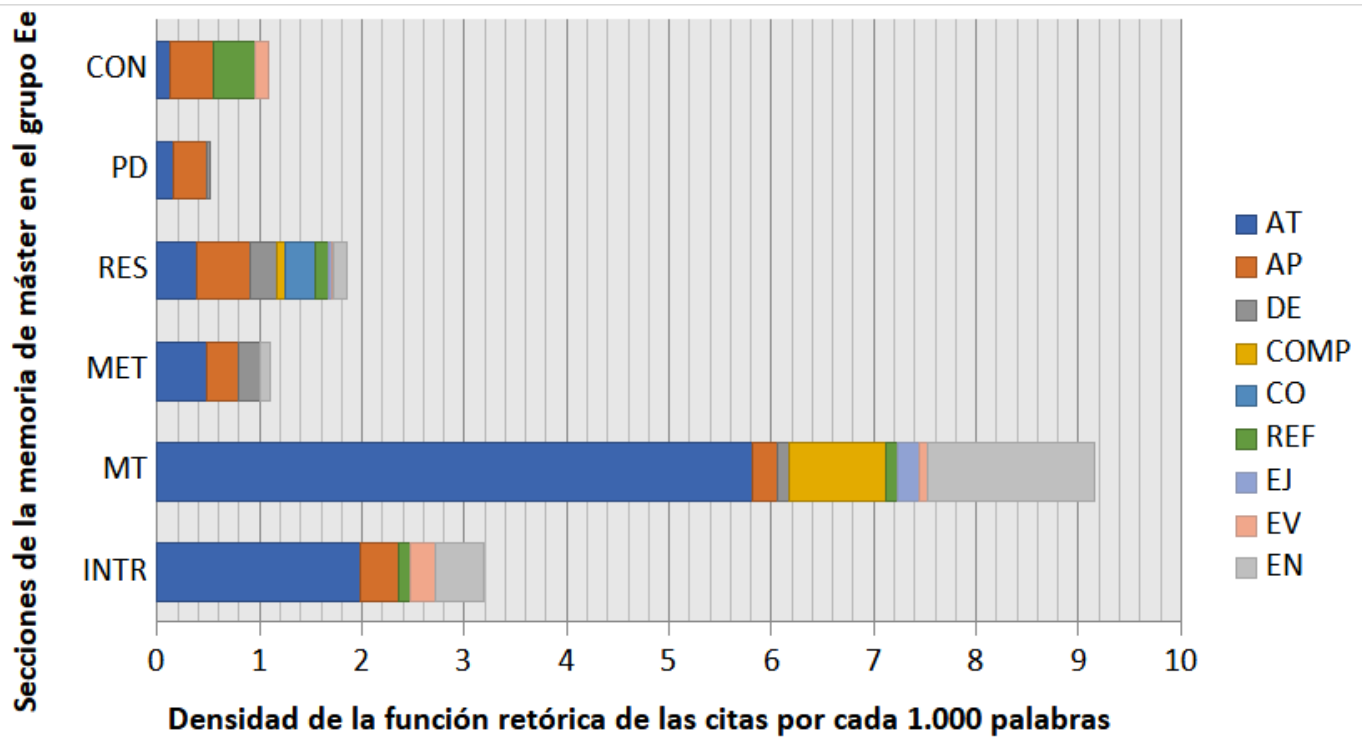


Figura 4: Densidad de las funciones retóricas de las citas por sección en el grupo Ee.

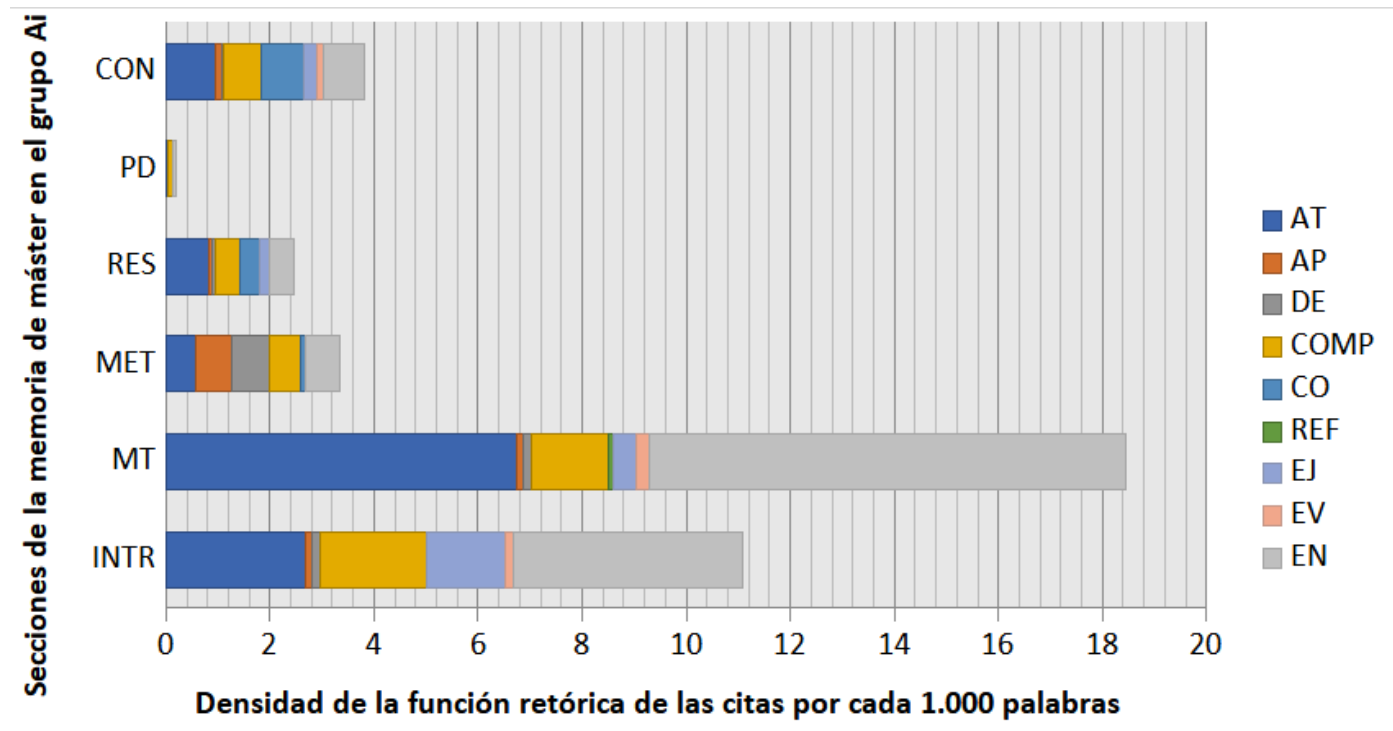

Figura 5: Densidad de las funciones retóricas de las citas por sección en el grupo Ai.

Como también se observaba en la Figura 5, las memorias redactadas por escritores españoles apenas obtienen ocurrencias en las secciones INTR $(3,19)$ y CON $(1,08)$ si se comparan con los autores de nacionalidad estadounidense, quienes registran respectivamente cifras de 11,17 y 3,85 citas por cada 1.000 palabras en estos apartados. Además de esta diferencia, los informantes de Ee recurren frecuentemente en INTR al uso de la función AT $(1,98=62,1 \%)$, cuando la distribución de las citas en Ai refleja un reparto más equitativo entre los grupos con EN (4,40=39,7\%), AT $(2,67=24,1 \%)$ y COMP $(2,04=18,4 \%)$, incidiendo en el contraste de ideas con otros autores en el texto. A diferencia de Ai $(0,15)$, en el grupo español destaca la presencia de la función $\operatorname{EV}(0,24)$, lo que muestra una ligera tendencia en este grupo a realizar comentarios valorativos sobre las fuentes citadas en este apartado. Por su parte, Ai prefiere utilizar las citas para ejemplificar los contenidos que presenta en esta sección con EJ $(1,52)$, más abundante que en el grupo Ee, que no registra ninguna ocurrencia en esta categoría.

En el apartado CON, la diferencia cuantitativa entre los grupos sobre las funciones utilizadas también resulta evidente y significativa, aunque esto no llegue a reflejarse en el porcentaje intragrupal contrastado de los subcorpus estudiados. Si bien las citas computadas en Ai en esta sección comprenden el 9,73\% del total, con una densidad de 3,85, en Ee llegan al 6,5\%, con 1,08. En cuanto a las categorías de las citas halladas en las memorias de Ee en CON, sobresalen AP $(0,41)$, REF $(0,41)$, EV $(0,13)$ y AT $(0,13)$, mientras que los autores estadounidenses utilizan con mayor frecuencia AT $(0,95), \mathrm{CO}(0,81)$ y EN $(0,77)$. De estos datos se colige un mayor interés por parte de los escritores españoles por comentar y evaluar el resultado de los trabajos con los que se 
compara su investigación. Por otro lado, mediante CO, el grupo Ai contrasta los datos obtenidos en su estudio con los resultados de los trabajos que han abordado previamente el tema de su investigación. Un factor que también influye en el predominio de $\mathrm{CO}$ en $\mathrm{CON}$ en el grupo Ai fue propiciado por el hecho de combinar los apartados de Discusión y Conclusiones en algunas de las memorias analizadas dentro de este subcorpus, en lugar de agrupar la sección de Conclusiones con la de Resultados (§ 3.1.).

En MT, se observa de nuevo la tendencia ya comentada en INTR sobre el reparto de las funciones retóricas de las citas en esa sección. En el grupo español destaca el empleo de AT $(5,82)$, allí donde las funciones de las citas realizadas por Ai presentan una mayor variación en el uso, identificándose referencias del tipo EN $(9,15)$, AT $(6,74)$, COMP $(1,49)$ y EJ $(0,45)$. Como ocurría también en INTR, en MT, los estudiantes de máster estadounidenses hacen un empleo más variado de las citas en este apartado, y a diferencia del grupo Ee -quienes utilizan mayormente la función AT para atribuir el conocimiento a la fuente-, los escritores norteamericanos tienden a contrastar los contenidos expuestos por los autores citados mediante el uso de EN. En MET y RES, los dos grupos parecen coincidir en el manejo de las mismas categorías, aunque cuantitativamente el uso de las citas en MET resulta superior en $\mathrm{Ai}(3,36)$ con respecto a $\mathrm{Ee}(1,11)$, hecho que indica la mayor relevancia otorgada por los primeros a atribuir a los autores citados la utilización de los instrumentos, métodos y conceptos prestados de otros autores en sus obras (DE: 0,75; AP: 0.69; EN: 0,66). En el conjunto de las memorias escritas en español, las categorías predominantes en MET son AT $(0,48)$, AP $(0,32)$ y DE $(0,21)$, por este orden de frecuencia. Sin embargo, en RES, donde la densidad de las citas alcanza cifras más parejas en ambos grupos (Ai: 2,49; Ee: 1,85 ), la variación en el reparto de las funciones de las citas es todavía una cuestión destacada. $\mathrm{Si}$ bien contienen prácticamente las mismas categorías (AT, $\mathrm{CO}$ y EN), la frecuencia varía significativamente en los casos de AT (Ai: 0,84; Ee: 0,39), CO (Ai: 0,37; Ee: 0,29) y EN (Ai: $0,47$; Ee: 0,13$)$. De entre todos estos datos, en Ee se observa un alto índice de la función $\operatorname{AP}(0,51)$ en la sección RES, quizás debido al desplazamiento de MET al apartado de RES en algunas de las memorias analizadas (§ 4.2.). En el subcorpus Ai, priman AT $(0,84), \mathrm{EN}(0,47), \operatorname{COMP}(0,47)$ y CO $(0,37)$, siguiendo la tendencia que ya se comentó para CON en el grupo Ai, pues algunas de las memorias combinaban la Discusión con RES, mientras que otras la mantenían dentro de la sección $\mathrm{CON}$, hecho que dificulta la comparación entre los grupos en estas secciones. En PD, las funciones AT $(0,16)$ y, sobre todo, AP $(0,32)$ fueron las más comunes en Ee. En el grupo Ai, únicamente un informante incluyó este apartado en su memoria, por lo que su uso de las citas resulta aleatorio y no significativo en la descripción de las funciones retóricas empleadas por su grupo en este apartado.

\subsection{Clasificación de los Resultados del Contraste entre los Grupos sobre la Frecuencia de las Funciones Retóricas de las Citas por Sección}

Los resultados relativos a la frecuencia de las funciones de las citas por cada 1.000 palabras en cada sección se ordenan en la Tabla 3 a modo de resumen. Para ello, se señalan tanto las funciones que han obtenido un mayor número de frecuencias $(+)$ por sección como las que menos $(-)$. Por lo tanto, la Tabla 3 indica la relación que se establece entre las funciones más utilizadas en cada apartado analizado de las memorias estudiadas en el corpus, cuya correlación sugiere que existe una serie de funciones típicas que se corresponden con una sección determinada.

En este sentido, en una investigación sobre las funciones de las citas en los apartados de la tesis doctoral escrita en inglés, Thompson (2001) comentaba que las distintas secciones cumplen propósitos muy específicos en este género, y que cada una de ellas se corresponde con un tipo de cita diferente que predomina de forma casi estandarizada en cada una de esas partes. Así, en MT, es esperable que se utilicen citas con una función claramente expositiva como, por ejemplo, AT, COMP y EN. También es de suponer que los estudiantes de máster harán uso en este apartado de las categorías REF y EJ, que sirven para ampliar y explicar las lecturas comentadas en el MT. Por otra parte, en el apartado de MET, las citas deberían ser más funcionales y hacer referencia a los 
materiales, instrumentos de análisis y conceptos de otros autores de los que se han servido como modelo para elaborar el capítulo de Metodología. Por ello, en esta sección, los escritores expertos tienden a utilizar las funciones DE y AP. En la sección de RES, debería haber un mayor predominio de la función $\mathrm{CO}$, que se usa para comparar los resultados del estudio con los resultados de las investigaciones precedentes realizadas en el mismo campo del saber, así como EV, usada con el fin de valorar el aporte de esos trabajos.

\begin{tabular}{|c|c|c|c|c|c|c|}
\hline & INTR & MT & MET & RES & PD & CON \\
\hline Grupos Ee+Ai (+) & $\begin{array}{l}\text { AT, EN, } \\
\text { COMP }\end{array}$ & $\begin{array}{l}\text { AT, EN, } \\
\text { COMP }\end{array}$ & $\begin{array}{c}\text { EN, AT, AP, } \\
\text { DE }\end{array}$ & $\begin{array}{c}\text { REF, AT, AP, } \\
\text { CO }\end{array}$ & $\begin{array}{c}\text { COMP, EN } \\
\text { AT }\end{array}$ & $\begin{array}{c}\text { AT, CO, EN, } \\
\text { COMP }\end{array}$ \\
\hline Grupo Ee (+) & $\mathrm{AT}, \mathrm{EN}, \mathrm{AP}$ & $\begin{array}{l}\text { AT, EN, } \\
\text { COMP }\end{array}$ & AT, AP, DE & $\begin{array}{c}\mathrm{AP}, \mathrm{AT}, \mathrm{CO}, \\
\mathrm{DE}\end{array}$ & $\mathrm{AP}, \mathrm{AT}, \mathrm{DE}$ & $\begin{array}{c}\text { AP/ REF, AT } \\
\text { EV }\end{array}$ \\
\hline Grupo Ai (+) & $\begin{array}{l}\text { EN, AT, } \\
\text { COMP }\end{array}$ & $\begin{array}{l}\text { EN, AT, } \\
\text { COMP }\end{array}$ & DE, AP, EN & $\begin{array}{c}\text { AT, EN/ } \\
\text { COMP, CO }\end{array}$ & $\begin{array}{c}\text { EN, COMP, } \\
\text { AT }\end{array}$ & $\mathrm{AT}, \mathrm{CO}, \mathrm{EN}$ \\
\hline Grupos Ee+Ai (-) & $\mathrm{EV}, \mathrm{DE}, \mathrm{CO}$ & $\begin{array}{l}\text { EV, DE, } \\
\text { REF, CO }\end{array}$ & $\mathrm{EV} / \mathrm{REF} / \mathrm{EJ}$ & DE, EJ, EV & $\mathrm{REF} / \mathrm{EJ} / \mathrm{EV}$ & $\mathrm{EJ} / \mathrm{EV}, \mathrm{DE}$ \\
\hline Grupo Ee (-) & $\begin{array}{c}\mathrm{DE} / \mathrm{COMP} / \\
\mathrm{EJ} / \mathrm{CO}\end{array}$ & $\mathrm{DE}, \mathrm{EV}, \mathrm{CO}$ & $\begin{array}{c}\mathrm{COMP} / \mathrm{REF} / \\
\mathrm{CO} / \mathrm{EJ} / \mathrm{EV}\end{array}$ & $\begin{array}{c}\text { COMP, EJ, } \\
\text { EV }\end{array}$ & $\begin{array}{c}\mathrm{COMP} / \mathrm{REF} / \\
\mathrm{EJ} / \mathrm{CO} / \mathrm{EV} / \\
\mathrm{EN}\end{array}$ & $\begin{array}{c}\mathrm{DE} / \mathrm{COMP} / \\
\mathrm{EN} / \mathrm{CO}\end{array}$ \\
\hline Grupo Ai (-) & $\begin{array}{c}\mathrm{AP} / \mathrm{DE} / \mathrm{EV}, \\
\mathrm{CO}, \mathrm{REF}\end{array}$ & AP, REF, CO & $\mathrm{REF} / \mathrm{EJ} / \mathrm{EV}$ & $\begin{array}{l}\text { AP, DE, } \\
\text { REF/ EV }\end{array}$ & $\begin{array}{l}\text { REF/ EJ/EV/ } \\
\text { AP, CO, DE }\end{array}$ & $\begin{array}{c}\mathrm{EV} / \mathrm{AP}, \mathrm{DE}, \\
\mathrm{REF}\end{array}$ \\
\hline
\end{tabular}

Tabla 3: Comparación entre los datos de las funciones retóricas de las citas con mayor y menor densidad por sección en las memorias escritas en español.

Los resultados de la distribución de las funciones retóricas de las citas por sección obtenidos en nuestro estudio convienen con esta descripción en MT, MET y RES, pues son los apartados más regulares en los que las categorías utilizadas coinciden más entre los grupos estudiados. Por el contrario, se advierte una mayor variabilidad en el resto de los aparatados entre los escritores que componen sus memorias en español y en inglés. Estas diferencias se aprecian igualmente en MT -aunque en menor medida que en estas otras secciones-, donde las cifras de las funciones utilizadas en las memorias escritas en lengua inglesa varían sustancialmente con respecto a Ee. En estas, se apunta a un mayor énfasis en el contraste de las ideas y un mayor esfuerzo en la transformación del conocimiento mediante el empleo de funciones dialógicas tales como EN y COMP, en contraste con el uso expositivo de AT, que cuenta con una presencia tan destacada en el grupo Ee. Coincidiendo con esta tendencia, otros autores que han investigado las funciones retóricas de las citas han sugerido que esta es la categoría que suelen utilizar principalmente los estudiantes inexpertos de posgrado, ya que sirve para atribuir el conocimiento a un autor en la exposición de los contenidos teóricos expuestos en el trabajo de investigación sin polemizar con sus observaciones (Petrić 2007; Samraj 2008, 2013; Mansourizadeh/Ahmad 2011; Soler-Monreal/ Gil-Salom, 2011; Sánchez-Jiménez 2013, 2018a; Venegas et al. 2013). Mediante su empleo, los escritores novatos se esfuerzan por dar la impresión de conocer convenientemente la bibliografía sobre el tema estudiado con el objetivo final de convencer al Tribunal de la validez y suficiencia de su investigación en la disciplina. De este modo, este apartado de MT, que comprende el mayor volumen de citas de la memoria, sirve como ejemplo para observar el comportamiento diferenciado de los grupos descritos, pues los informantes que han recibido formación en escritura académica (Ai) tienden a hacer un uso más variado y complejo de las citas en este apartado. Por el 
contrario, vemos que los textos producidos por Ee se articulan en torno a la categoría AT en esta misma sección, con la función primordial de exponer el conocimiento adquirido a través de las fuentes citadas en sus escritos (INTR, MT, MET, RES, PD), a diferencia de Ai, quienes prefieren contrastar los distintos puntos de vista aportados por los autores referidos en las fuentes mediante la función EN (INTR, MT, MET, RES, PD, CON). Del mismo modo, en el subcorpus de Ai, la alusión a generalizaciones (categoría COMP) relacionadas con el conocimiento establecido en la disciplina ocurre con mayor amplitud y frecuencia en secciones como INTR, MT, RES o PD, cuando en Ee su uso solo destaca en MT.

Por el contrario, las referencias a los conceptos teóricos prestados de otros autores son más frecuentes en el grupo español, quienes emplean AP de manera reiterada en INTR, MET, RES, PD o CON. También son definidoras de este subcorpus Ee las remisiones a las fuentes citadas en CON para ampliar la información expuesta en este apartado mediante REF. Por su parte, los estudiantes de Ai coinciden en el uso frecuente de la función CO en esta parte de la memoria de máster. Aunque esta no es la categoría con mayor densidad de citas en el grupo, sí posee una presencia destacada en la sección, cuyo propósito consiste en comparar los resultados de la propia investigación con los obtenidos por otros autores y promocionar así su escrito entre la comunidad discursiva. Otro rasgo que individualiza al grupo Ai es la mención más frecuente en MET de la procedencia de los paradigmas y taxonomías de las investigaciones previas en las que se basa su estudio, haciendo para ello un uso habitual de esta sección.

En general, en nuestro trabajo se observa que en este estadio preliminar de la investigación universitaria de posgrado -en el que los alumnos aún están comenzando su camino hacia su profesionalización como investigadores- todavía no se dominan las convenciones retóricas y discursivas del género. Así, por ejemplo, frente al predominio de funciones más expositivas como AT, EN y COMP en la mayor parte de las secciones analizadas en el corpus (siendo AT la más expositiva de las tres), sorprende la baja frecuencia de la categoría EV en $\operatorname{RES}(0,01)$ y CON $(0,06)$, apartados en los que comúnmente los escritores expertos exponen sus juicios y valoraciones sobre otros trabajos relacionados con su investigación al contrastarlos con los resultados obtenidos en su estudio. Esta ausencia se repite en MT, lo que confirma la falta de posicionamiento de la voz de estos autores noveles, quienes prefieren adoptar una cierta invisibilidad al saberse en situación de asimetría con respecto a los autores expertos que citan en sus trabajos de posgrado y con los miembros del Tribunal que van a juzgar sus escritos.

Por otra parte, a pesar del uso masivo de funciones de tipo expositivo (AT, EN y COMP) que predominan en los textos del corpus, también se recogen en este numerosos casos que muestran cómo los estudiantes comienzan a asimilar con criterio algunas de las convenciones discursivas establecidas en la investigación académica con respecto al empleo de las citas. De este modo, en el apartado MET, se utilizan las funciones DE y AP de manera frecuente con el fin de referenciar conceptos teóricos y paradigmas prestados de otros autores para construir su propia investigación. Otra categoría destacada en la sección CON es CO, la cual se utiliza acertadamente con elevada frecuencia para comparar los resultados de sus estudios con otros precedentes realizados sobre el tema investigado.

\section{Conclusiones}

El propósito de la presente investigación partía de la comparación de las funciones retóricas que se adscriben a las citas utilizadas en cada apartado del trabajo final de máster por un grupo de estudiantes españoles que escriben en su lengua nativa con las usadas por escritores estadounidenses en inglés, con el fin de conocer si presentan diferencias significativas entre sí. Los datos referidos a la densidad de las citas por sección revelaron que el apartado que presentaba un mayor número de citas era MT, el cual concentraba junto a INTR el $74 \%$ de la densidad total de las citas computadas. Estas elevadas frecuencias reflejan que el género analizado tiene un marcado carácter expositivo, pues en él los escritores tratan de contextualizar su estudio y 
demostrar ante la comunidad académica -representada por el Tribunal de máster- que dominan los contenidos sobre el tema objeto de investigación.

En el análisis individualizado de los apartados que componen la memoria, se ha hallado un dato relevante en relación con las secciones INTR y CON, en las cuales la frecuencia de las citas en el grupo Ai es muy superior a la registrada por el grupo Ee. Esto parece deberse a los valores promocionales comprendidos en las secciones redactadas en inglés, como confirman los datos expuestos en estudios de características similares (Fløttum et al. 2006; Mur Dueñas 2009; SolerMonreal/Gil-Salom 2011; Soler-Monreal et al. 2011).

Del predominio de unas determinadas funciones retóricas de las citas típicas de cada sección sobre otras menos habituales, se concluye que existe una serie de categorías que se repiten de manera frecuente en cada apartado (INTR [AT, EN, COMP], MT [AT, EN, COMP], MET [EN, AT, AP, DE], RES [REF, AT, AP, CO], PD [COMP, EN, AT], CON [AT, CO, EN, COMP]). La coincidencia de un conjunto de funciones con el propósito retórico de cada sección evidencia una estrecha vinculación entre la cita y la organización estructural del texto académico. Esta asociación no es siempre estable entre los grupos analizados, pues está sometida a la variación de los patrones retórico-culturales, aunque el predominio de AT en la mayoría de las secciones de la memoria de máster es común a ambos. No obstante, el objetivo principal de este género discursivo es el de probar la suficiencia investigadora del estudiante mediante la demostración del dominio de los conceptos clave y el manejo de las convenciones discursivas de la disciplina. AT es también la categoría que destaca por su alta frecuencia de aparición en la mayoría de las secciones de los textos analizados en el subcorpus Ee, pues como bien refiere Vázquez (2001: 14), la abundancia de este tipo de cita es un rasgo distintivo de los trabajos académicos de los investigadores españoles en relación con otras culturas. Sin embargo, a diferencia de lo que ocurre con Ee, en el subcorpus Ai se impone un valor más promocional de las citas en las memorias escritas en lengua inglesa mediante el uso más frecuente de las funciones EN y COMP, que sirven respectivamente para establecer contrastes entre los conceptos y los autores más destacados mencionados en la bibliografía, así como para resaltar los trabajos fundamentales que sirven de referencia en la disciplina.

En resumen, con este estudio se ha verificado que sí existen diferentes convenciones culturales influidas por los patrones retóricos, culturales y educativos adquiridos por los estudiantes de posgrado, las cuales caracterizan el uso que estos hacen de las funciones retóricas de las citas. Dichas convenciones se manifiestan en nuestro estudio en la estructura que se utiliza para vertebrar la memoria de máster y en el tipo de citas incluidas en cada sección. Los datos que arroja el análisis de los resultados muestran que esta variación se corresponde con una distinción en el grado de la instrucción formal que reciben los informantes estadounidenses y los españoles, siendo así la formación en cada cultura una de las variables principales que incide significativamente en los resultados obtenidos en la investigación. De este modo, los estudiantes que han recibido una formación específica en escritura académica en trabajos de investigación a nivel universitario, los estadounidenses, presentan datos más homogéneos tanto en la organización de la superestructura del trabajo fin de máster como en las prácticas retórico-discursivas de las funciones de las citas empleadas en los diferentes apartados del género analizado. Los informantes de Ee, por su parte, no adoptan los mismos patrones retóricos que los escritores norteamericanos en sus textos y se distancian parcialmente de las convenciones dominantes en la disciplina de la Lingüística Aplicada en torno a la elaboración de trabajos de investigación científica. De igual modo, se constata el efecto de esta variable en la organización superestructural más irregular de las secciones de la memoria de máster en este grupo, la cual presenta ciertas señas de anarquía que contrastan con los textos más homogéneos elaborados en lengua inglesa.

Sobre las limitaciones encontradas en el estudio, quizás la más relevante sea la excesiva variabilidad en la estructura de los trabajos seleccionados, que interfiere de manera significativa en los resultados de la investigación. Por ejemplo, la memoria analizada de Ai5 pone en entredicho la validez de los datos obtenidos en la sección PD, debido a que solo este texto acumula el mayor 
porcentaje de la densidad de las citas sobre el total en su grupo, por ser el único escritor que utiliza este apartado. Por otro lado, la inconsistencia de los informantes en la división que hacen de los apartados también ha dificultado el cómputo parcial de las citas por sección, especialmente en los casos de Metodología, Resultados, Discusión y Conclusiones, los cuales tienden a fusionarse unos con otros de forma aleatoria en los textos de ambos grupos. En próximos estudios sobre este tema, deberían seleccionarse memorias que faciliten la comparación de estos resultados teniendo en cuenta este aspecto. Del mismo modo, también habría que ampliar el corpus para favorecer la generalización de los resultados. Sin embargo, la vasta extensión de este género justifica en muchas ocasiones la elección de corpus más reducidos de entre 6 y 15 obras, como en este caso, en el que partimos del corpus compilado para un estudio previo (Sánchez-Jiménez 2018a). Es por este motivo que los estudios realizados sobre las funciones de las citas en los textos de posgrado, tanto sobre la memoria de máster (p. ej. Petrić 2007; Samraj 2008, 2013; Jalilifar 2012; Rabab'ah/Ahmed Al-Marshadi 2013; Sánchez-Jiménez 2013, 2018a; Venegas et al. 2013; Nguyen/Pramoolsook 2015) como de la tesis doctoral (p. ej. Dong 1996; Thompson 2001; Thompson/Tribble 2001; Gallardo 2010; Soler-Monreal/Gil-Salom, 2011), se caracterizan por analizar un número reducido de textos. Las consecuencias, como ocurre en nuestra investigación con la sección PD, es que la sustitución de un par de memorias por otras en el estudio tiene efectos relevantes en el análisis de los datos.

En conclusión, pensamos que las aproximaciones empíricas desde la Retórica Intercultural a este aspecto pragmalingüístico del discurso, como se ha mostrado a lo largo de este artículo, resultan útiles para comprender mejor la variabilidad que afecta a los patrones retóricos del discurso escrito en distintas lenguas. Asimismo, a partir de las diferencias señaladas en los resultados del estudio entre ambas culturas, se podrá establecer en el futuro una didáctica (Sánchez-Jiménez 2018b) que permita mejorar los trabajos académicos elaborados por los estudiantes universitarios nativos (L1) y extranjeros (L2) inscritos en los niveles de posgrado que escriben su memoria de máster en inglés o español como L2 con el fin de adaptarse a estas convenciones culturales en el uso de las citas. Así, esperamos que las conclusiones expuestas en el presente trabajo inspiren nuevas investigaciones que profundicen en estas cuestiones y que su aplicación sirva en el futuro como un marco pedagógico en cursos de formación. Esto contribuirá, sin duda alguna, a la mejora de los trabajos académicos de investigación elaborados por los estudiantes universitarios en los niveles de segundo y tercer ciclo.

\section{Referencias}

Beke, Rebecca 2008: El discurso académico: la atribución del conocimiento en la investigación educativa. En Núcleo 25, 13-35.

Bhatia, Vijay 1993: Analysis Genre: Language Use in Professional Settings. Londres: Longman.

Bunton, David 2002: Generic Moves in PhD Thesis Introductions. En Flowerdew, John (ed.), Academic Discourse. London: Longman, 57-75.

Carbonell-Olivares, María/Gil-Salom, Luz/Soler-Monreal, Carmen 2009: The Schematic Structure of Spanish PhD Thesis Introductions. En Spanish in Context 6(2), 151-176.

Connor, Ulla 1996: Contrastive Rhetoric. Cross-cultural Aspects of Second Language Writing. Cambridge: Cambridge University Press.

Connor, Ulla 2002: New Directions in Contrastive Rhetoric. En TESOL Quarterly 36(4), 493-510.

Connor, Ulla 2011: Intercultural Rhetoric in the Writing Classroom. Ann Arbor, MI: The University of Michigan Press.

Dong, Yu Ren 1996: Learning How to Use Citations for Knowledge Transformation: Non-native Doctoral Student's Dissertation Writing in Science. En Research in the Teaching of English 30(4), 428-457.

Dontcheva-Navratilova, Olga 2016: Rhetorical Functions of Citations in Linguistics Research Articles: A Contrastive (English-Czech) study. En Discourse and Interaction 9(2), 51-74.

Dudley-Evans, Tony 1986: Genre Analysis: An Investigation of the Introduction and Discussion Sections of MSc Dissertations. En Coulthard, Malcolm (ed.), Talking About Text. English Language Research. Birmingham: University of Birmingham, 128-145. 
Dudley-Evans, Tony 1994: Genre Analysis: An Approach for Text Analysis for ESP. En Coulthard, Malcolm (ed.), Advances in Written Text Analysis. London: Routledge, 219-228.

Dudley-Evans, Tony 2000: Genre Analysis: A Key to a Theory of ESP? En Ibérica 2, 3-11.

Fløttum, Kjersti/Dahl, Trine/Kinn, Torodd 2006: Academic Voices: Across Languages and Disciplines. Amsterdam/ Philadelphia: John Benjamins.

Flowerdew, John 2002: Academic Discourse. Londres: Longman.

Gallardo, Susana 2010: La citación en tesis doctorales de biología y lingüística. En Ikala 15(26), 153-177.

Grabe, William/Kaplan, Robert B. 1996: Theory and Practice of Writing. London: Longman.

Harwood, Nigel 2009: An Interview-based Study of the Functions of Citations in Academic Writing Across Two Disciplines. En Journal of Pragmatics 41(3), 497-518.

Harwood, Nigel/Petrić, Bojana 2012: Performance in the Citing Behavior of Two Student Writers. Written Communication 29 (I), 55-103.

Hewings, Ann/ Lillis, Theresa/Vladimirou, Dimitra 2010: Who's Citing Whose Writings? A Corpus Base Study of Citations as Interpersonal Resource in English Medium National and English Medium International Journals. En Journal of English for Academic Purposes 9(2), 102-115.

Hopkins, Andy/Dudley-Evans, Tony 1988: A genre-based investigation of the discussion sections in articles and dissertations. En English for Specific Purposes 7, 113-22.

Hyland, Ken 1999: Academic Attribution: Citation and the Construction of Disciplinary Knowledge. En Applied Linguistics 20(3), 341-367.

Hyland, Ken 2000: Disciplinary Discourses: Social Interactions in Academic Writing. Harlow, UK: Longman.

Hyland, Ken 2002: Authority and Invisibility: Authorial Identity in Academic Writing. En Journal of Pragmatics 34, 1091-1112.

Hyland, Ken 2005: Stance and engagement: A model of interaction in academic discourse. En Discourse Studies 7(2), 173-192.

Hyland, Ken 2017: Metadiscourse: What is it and Where is it Going? En Journal of Pragmatics 113, 16-29.

Jalilifar, Alireza 2012: Academic Attribution: Citation Analysis in Master's Theses and Research Articles in Applied Linguistics. En International Journal of Applied Linguistics 22(1), 23-41.

Kaplan, Robert B. 1966: Cultural Thought Patterns in Inter-cultural Education. En Language Learning 16(1-2), 1-20.

Kaplan, Robert B. 1978: Contrastive Rhetoric: Some Hypotheses. En ITL 39-40, 61-72.

Kinkead, Joyce 2001: The National Writing Centers Association as Mooring: A Personal History of the First Decade. En Barnett, Robert W./ Blumner, Jacob S. (eds..), The Allyn and Bacon Guide to Writing Center Theory and Practice. Boston: Allyn and Bacon, 29-40.

Leki, Ilona 1991: Twenty-Five Years of Contrastive Rhetoric: Text Analysis and Writing Pedagogies. En TESOL Quarterly 25(1), 123-143.

Lux, Paul/Grabe, William 1991: Multivariate Approaches to Contrastive Rhetoric. En Lenguas Modernas 18, 133-60.

Mansourizadeh, Kobra/Ahmad, Ummul 2011: Citation Practices Among Non-native Expert and Novice Scientific Writers. En Journal of English for Academic Purposes 10, 152-161.

Mayor Serrano, Blanca 2004: La citación en el artículo de divulgación médica (inglés-español) y su importancia en la formación de traductores. Panace@ 5(17-18), 255-259.

Mayor Serrano, Blanca 2006: La citación en la comunicación médica escrita (inglés-español): funciones y tipos. En Lebende Sprachen 51(2), 72-78.

Montaño-Harmon, María Rosario 1991: Discourse Features of Written Mexican Spanish: Current Research in Contrastive Rhetoric and Its Implications. En Hispania 74, 417-425.

Mur Dueñas, Pilar 2009: Citation in Business Management Research Articles: A Contrastive (English-Spanish) Corpus-based Analysis. En Suomela-Salmi, Eija/Dervin, Fred (eds.), Cross-Linguistic and Cross-Cultural Perspectives on Academic Discourse. Amsterdam: John Benjamins, 49-60.

Nguyen, Thi Thuy Loan/Pramoolsook, Issra 2015: Citation in Vietnamese TESOL: Analysis of Master's Thesis Introduction Chapters. En The Asian ESP Journal 11(1), 95-120.

Nicolaisen, Jeppe 2007: Citation Analysis. En Annual Review of Information Science and Technology 41(1), 609-641.

Petrić, Bojana 2007: Rhetorical Functions of Citations in High and Low-rated Master's Thesis. En Journal of English for Academic Purposes 6, 238-253.

Rabab'ah, Ghaleb/Al-Marshadi, Ahmed 2013: Integrative vs. Non-Integrative Citations Among Native and Nonnative English Writers. En International Education Studies 6(7), 78-87. 
Reid, Joy 1992: A Computer Text Analysis of Four Cohesion Devices in English Discourse by Native and Non-native Writers. En Journal of Second Language Writing 1(2), 79-107.

Samraj, Betty 2008: A Discourse Analysis of Master's Theses Across Disciplines with a Focus on Introductions. En Journal of English for Academic Purposes 7, 55-67.

Samraj, Betty 2013: Form and Function of Citations in Discussion Sections of Master's Theses and Research Articles. En Journal of English for Academic Purposes 12, 299-310.

Sánchez Jiménez, David 2011: Las funciones retóricas de la citación en la escritura académica universitaria. Estudio comparado del género de memorias de máster en nativos españoles y estudiantes filipinos en ELE. Trabajo de investigación D.E.A. Madrid.

Sánchez-Jiménez, David 2012: El uso de las funciones de las citas en la estructura retórica de las Introducciones de memorias de máster escritas en español por estudiantes nativos españoles y filipinos no nativos. En Revista Nebrija de Lingüística Aplicada a la Enseñanza de Lenguas 12, 137-187.

Sánchez-Jiménez, David 2013: Aplicabilidad de la tipología de las funciones retóricas de las citas al género de la memoria de máster en un contexto transcultural de enseñanza universitaria. En Revista Signos 46(81), 82-104.

Sánchez-Jiménez, David 2015: 50 años de evolución en los estudios lingüísticos transculturales: de la Retórica Contrastiva a la Retórica Intercultural. En Argus-a. Artes \& Humanidades V(18).

Sánchez-Jiménez, David 2018a: Estudio contrastivo intercultural de las funciones retóricas de las citas: la producción de textos académicos en el nivel de posgrado escritos en español e inglés. En Pragmalingüística 26, 373-392.

Sánchez-Jiménez, David 2018b: Aproximación didáctica al apendizaje de las funciones retóricas de las citas en el discurso académico universitario. En van Hooft, Andreu (Coord.), El Español como lengua para la innovación profesional. Bruselas: Consejería de Educación, 54-68.

Sánchez-Jiménez, David 2018c: Elementos persuasivos de la citación en el discurso académico de posgrado: los verbos introductores de las citas en la sección de la Conclusión. En González Peláez, Marta/ Valderrama Santomé, Mónica (eds.), Discursos Comunicativos Persuasivos Hoy. Madrid: Tecnos, 415-428.

Santiago, Ramón Luis 1970: A contrastive analysis of some rhetorical aspects in the writing in Spanish and English of Spanish-speaking college students in Puerto Rico. En Dissertation Abstracts International 31(12), 6368A.

Soler-Monreal, Carmen/Gil-Salom, Luz 2011: A cross-language study on citation practice in PhD thesis. En International Journal of English Studies 11(2), 53-75.

Soler-Monreal, Carmen/ Gil-Salom, Luz 2014: Academic voices and claims. Reviewing practices in research writing. En Gil-Salom, Luz/Soler-Monreal, Carmen (eds.), Dialogicity in Written Specialised Genres. Amsterdam: John Benjamins, 23-54.

Soler-Monreal, Carmen/Carbonell-Olivares, María/Gil-Salom, Luz 2011: A contrastive study of the rhetorical organisation of English and Spanish PhD thesis introductions. En English for Specific Purposes 30, 4-17.

Swales, John 1986: Citation Analysis and Discourse Analysis. En Applied Linguistics 7(1), 39-56.

Swales, John 1990: Genre Analysis: English in Academic and Research Settings. Cambridge, England and New York: Cambridge University Press.

Swales, John 2004: Research Genres: Explorations and Applications. Cambridge: Cambridge University Press.

Swales, John/Feak, Christine 1994: Academic Writing for Graduate Students. Ann Arbor: University of Michigan Press.

Thompson, Geoff/Ye, Yiyun 1991: Evaluation in the Reporting Verbs Used in Academic Papers. En Applied Linguistics 12(4), 365-382.

Thompson, Paul 2001: A pedagogically-motivated Corpus-based Examination of PhD Theses: Macroestructure, Citation Practices and Uses of Modal Verbs. Tesis doctoral (PhD). Reading: University of Reading.

Thompson, Paul 2005: Points of Focus and Position Intertextual Reference in PhD Theses. En Journal of English for Academic Purposes 4, 307-323.

Thompson, Paul/Tribble, Chris 2001: Looking at Citations: Using Corpora in English for Academic Purposes. En Language Learning \& Technology 5(3), 91-105.

Vázquez, Graciela (Coord.) 2001: Guía didáctica del discurso académico escrito: ¿cómo se escribe una monografía? Madrid: Edinumen.

Venegas. René/Meza, Paulina/Martínez Hincapié, Juan 2013: Procedimientos discursivos en la atribución del conocimiento en tesis de lingüística y filosofía en dos niveles académicos. En Revista de Lingüística Teórica y Aplicada 51(1), 153-179.

Wette, Rosemary 2010: Evaluating Student Learning in a University-level EAP Unit on Writing Using Sources. En Journal of Second Language Writing 19, 158-177. 
White, Howard D. 2004: Citation Analysis and Discourse Analysis Revisited. En Applied Linguistics 25(1), 89-116.

\section{Anexo I}

\begin{tabular}{|c|c|c|c|c|c|c|c|c|c|c|c|}
\hline Ee & AT & AP & DE & COMP & CO & REF & EJ & EV & EN & $\begin{array}{c}\text { Total } \\
\text { suma }\end{array}$ & $\mathbf{\%}$ \\
\hline INTR & 1,98 & 0,37 & 0 & 0 & 0 & 0,12 & 0 & 0,24 & 0,48 & $\mathbf{3 , 2 2}$ & $\mathbf{1 8 , 8}$ \\
MT & 5,82 & 0,24 & 0,11 & 0,94 & 0 & 0,13 & 0,20 & 0,09 & 1,62 & $\mathbf{9 , 2 1}$ & $\mathbf{5 3 , 8}$ \\
MET & 0,48 & 0,32 & 0,21 & 0 & 0 & 0 & 0 & 0 & 0,10 & $\mathbf{1 , 1 4}$ & $\mathbf{6 , 6}$ \\
RES & 0,39 & 0,51 & 0,26 & 0,09 & 0,29 & 0,13 & 0,04 & 0,01 & 0,13 & $\mathbf{1 , 9 0}$ & $\mathbf{1 1 , 2}$ \\
PD & 0,16 & 0,32 & 0,03 & 0 & 0 & 0 & 0 & 0 & 0 & $\mathbf{0 , 5 3}$ & $\mathbf{3 , 1}$ \\
CON & 0,13 & 0,41 & 0 & 0 & 0 & 0,41 & 0 & 0,13 & 0 & $\mathbf{1 , 1 1}$ & $\mathbf{6 , 5}$ \\
Total & $\mathbf{2 , 7 5}$ & $\mathbf{0 , 3 5}$ & $\mathbf{0 , 1 4}$ & $\mathbf{0 , 4 3}$ & $\mathbf{0 , 0 8}$ & $\mathbf{0 , 1 1}$ & $\mathbf{0 , 1 0}$ & $\mathbf{0 , 0 5}$ & $\mathbf{0 , 7 6}$ & & \\
\% & $\mathbf{5 7 , 3}$ & $\mathbf{7 , 5}$ & $\mathbf{2 , 9}$ & $\mathbf{8 , 9}$ & $\mathbf{1 , 7}$ & $\mathbf{2 , 4}$ & $\mathbf{2 , 2}$ & $\mathbf{1 , 2}$ & $\mathbf{1 5 , 9}$ & & \\
PRO & $\mathbf{1 , 4 9}$ & $\mathbf{0 , 3 6}$ & $\mathbf{0 , 1 0}$ & $\mathbf{0 , 1 7}$ & $\mathbf{0 , 0 4}$ & $\mathbf{0 , 1 3}$ & $\mathbf{0 , 0 4}$ & $\mathbf{0 , 0 7}$ & $\mathbf{0 , 3 8}$ & & \\
DEM & $\mathbf{2 , 2 2}$ & $\mathbf{0 , 0 9}$ & $\mathbf{0 , 1 1}$ & $\mathbf{0 , 3 7}$ & $\mathbf{0 , 1 1}$ & $\mathbf{0 , 1 4}$ & $\mathbf{0 , 0 8}$ & $\mathbf{0 , 0 9}$ & $\mathbf{0 , 6 2}$ & & \\
\hline
\end{tabular}

Tabla 4: Densidad de las funciones retóricas de las citas en el grupo Ee.

\section{Anexo II}

\begin{tabular}{|c|c|c|c|c|c|c|c|c|c|c|c|}
\hline Ai & AT & AP & DE & COMP & CO & REF & EJ & EV & EN & $\begin{array}{c}\text { Total } \\
\text { suma }\end{array}$ & $\mathbf{\%}$ \\
\hline INTR & 2,67 & 0,15 & 0,15 & 2,04 & 0 & 0 & 1,52 & 0,15 & 4,40 & $\mathbf{1 1 , 1 7}$ & $\mathbf{2 1 , 7}$ \\
MT & 6,74 & 0,13 & 0,15 & 1,49 & 0 & 0,08 & 0,45 & 0,25 & 9,15 & $\mathbf{1 8 , 4 8}$ & $\mathbf{3 5 , 8}$ \\
MET & 0,56 & 0,69 & 0,75 & 0,59 & 0.09 & 0 & 0 & 0 & 0,66 & $\mathbf{3 , 3 6}$ & $\mathbf{6 , 5}$ \\
RES & 0,84 & 0,06 & 0,05 & 0,47 & 0,37 & 0 & 0,21 & 0 & 0,47 & $\mathbf{2 , 4 9}$ & $\mathbf{4 , 8}$ \\
PD & 0,04 & 0 & 0 & 0,08 & 0 & 0 & 0 & 0 & 0,08 & $\mathbf{0 , 2}$ & $\mathbf{2 3 , 6}$ \\
CON & 0,95 & 0,13 & 0,04 & 0,72 & 0,81 & 0 & 0,26 & 0,13 & 0,77 & $\mathbf{3 , 8 5}$ & $\mathbf{7 , 6}$ \\
Total & $\mathbf{2 , 8 2}$ & $\mathbf{0 , 2 1}$ & $\mathbf{0 , 2 0}$ & $\mathbf{1 , 0 1}$ & $\mathbf{0 , 2 3}$ & $\mathbf{0 , 0 2}$ & $\mathbf{0 , 3 5}$ & $\mathbf{0 , 1 0}$ & $\mathbf{3 , 5 9}$ & & \\
\% & $\mathbf{3 3 , 0 5}$ & $\mathbf{2 , 4 6}$ & $\mathbf{2 , 3 4}$ & $\mathbf{1 1 , 8 4}$ & $\mathbf{2 , 6 9}$ & $\mathbf{0 , 2 3}$ & $\mathbf{4 , 1 1}$ & $\mathbf{1 , 1 7}$ & $\mathbf{4 2 , 1 1}$ & & \\
PRO & $\mathbf{2 , 4 2}$ & $\mathbf{0 , 2 4}$ & $\mathbf{0 , 1 9}$ & $\mathbf{1 , 6 1}$ & $\mathbf{0 , 3 1}$ & $\mathbf{0 , 0 1}$ & $\mathbf{0 , 4 0}$ & $\mathbf{0 , 0 8}$ & $\mathbf{3 , 2 5}$ & & \\
DEM & $\mathbf{2 , 3 2}$ & $\mathbf{0 , 2 3}$ & $\mathbf{0 , 2 8}$ & $\mathbf{1 , 4 8}$ & $\mathbf{0 , 3 4}$ & $\mathbf{0 , 0 3}$ & $\mathbf{0 , 5 7}$ & $\mathbf{0 , 1 0}$ & 3,38 & & \\
\hline
\end{tabular}

Tabla 5: Densidad de las funciones retóricas de las citas por lengua en el grupo Ai. 